\title{
Identification of Missense Mutation Pro77Arg As A Founder Mutation in Morquio-A Syndrome in Indian Gujarati Patel Ethnicity
}

\section{Jayesh J Sheth ( $\square$ jayesh.sheth@frige.co.in )}

FRIGE's Institute of Human Genetics https://orcid.org/0000-0001-5936-5192

Riddhi Bhavsar

FRIGE's Institute of Human Genetics

Aadhira Nair

FRIGE's Institute of Human Genetics

Chandni Patel

FRIGE's Institute of Human Genetics

Premal Naik

Rainbow Children's Orthopedic Centre

Maulin Shah

Ortho Kids Clinic

Mayank Chaudhary

Mayflower Women's Hospital

Frenny Sheth

FRIGE's Institute of Human Genetics

Nishtha Pandey

Centre for Human Genetics

Prajakta Deshpande

Centre for Human Genetics

Shweta Dubey

Centre for Human Genetics

Sreeja Nair

Centre for Human Genetics

Vandana Tamraparni

Centre for Human Genetics

Sudha Srinivasan

Centre for Human Geneticxs

Research article

Keywords: Mucopolysaccharidosis type IVA (MPS IVA), Morquio A syndrome, GALNS, GAG, founder mutation, skeletal dysplasia, p.P77R.

Posted Date: September 21st, 2020

DOI: https://doi.org/10.21203/rs.3.rs-65487/v1

License: (c) (i) This work is licensed under a Creative Commons Attribution 4.0 International License. Read Full License 


\section{Abstract}

Background: Morquio A syndrome (MPS IVA) is a mucopolysaccharide group storage disorder caused due to the deficient activity of the lysosomal enzyme N-acetylgalactoseamine-6-sulfatase encoded by GALNS. The present study represents the mutation spectrum of GALNS in 25 Gujarati Patel patients of India clinically and biochemically confirmed with Morquio-A disorder.

Methods: Urinary GAG quantitation and leucocyte enzyme assay was carried out in all 25 patients. This was followed by molecular characterization by amplification and sequencing of the exons and adjacent intronic regions of GALNS gene. Haplotype analysis was performed in patients showing p.P77R variant, using microsatellite markers D16S3121, D16S3026 and D16S3023 and SNPs.

Results: We identified 11 mutations that include eight missense mutations: (p.L36R, p.D39G, p.P77R, p.C79R, pP125L, p.P151L, p.G255A and p.L350P), one splice site mutation: (c.121-7C>G), one small insertion: (c.1241_1242insA, p.1416HfsTer2) and one small deletion: (c.839_41delACA). Of these, three missense mutations (p.D39G, p.G255A and p.L350P), one splice site and the two indels mentioned above are novel. In the present study, we found maximum number of mutant alleles in exon 2, and of note, the variant p.P77R was seen in fourteen patients.

Conclusion: p.P77R variant was predominantly found in Gujarati Patel community and the results of haplotype analysis indicated it to be the founder mutation in this community. Further, a study of 200 unrelated healthy control participants from Gujarat has identified this mutation in the heterozygous status in two individuals. Overall, our study suggests that p.P77R is likely to be a founder mutation for Morquio-A syndrome in Gujarati Patel ethnicity.

\section{Background}

Morquio- A syndrome, (MIM\# 253000) is an autosomal recessive glycosaminoglycan storage disorder with progressive skeletal dysplasia, among other symptoms. It is caused due to deficient activity of the lysosomal enzyme N-acetylgalactoseamine-6-sulfatase (GALNS, E.C.3.1.6.4) resulting from mutations in GALNS gene (OMIM* 612222) $(1,2)$. The deficiency of GALNS in Morquio-A syndrome patients results in the accumulation of glycosaminoglycans (GAG) such as keratan sulfate (KS) and chondroitin-6-sulfate (C6S) (3). The clinical features are characterized by a noticeable short stature, protrusion of the chest, kyphoscoliosis, genu valgum, laxity of joints and abnormal gait (4-6). Spine is predominantly involved in these patients (7), without affecting neuronal function.

This is a rare lysosomal storage disorder, with an estimated incidence ranging from one in 76,000 in Northern Ireland to one in 640,000 births in Western Australia $(4,8,9)$ and one in 450,000 in the Netherlands, and Portugal $(8,10)$. Though study in Asian population is scarce, the available estimates of birth prevalence include 1 in 500,000 live births in Japan, 1 in 304,000 in Taiwan (11), and 1 in 701,000 live births in Malaysia (12).

As of July 2020, 327 mutations in the GALNS have been reported with 242 as missense/nonsense mutations, 37 indels, 32 splicing site mutations, and 3 complex rearrangements (13). The ten most frequently reported GALNS alterations from patients with Morquio-A across the world are- c. $1156 \mathrm{C}>\mathrm{T}$, c. $337 \mathrm{~A}>\mathrm{T}, \mathrm{c} .901 \mathrm{G}>\mathrm{T}, \mathrm{c} .120+1 \mathrm{G}>\mathrm{A}, \mathrm{c} .1171 \mathrm{~A}>\mathrm{G}, \mathrm{c} .935 \mathrm{C}>\mathrm{G}, \mathrm{c} .871 \mathrm{G}>\mathrm{A}, \mathrm{c} .860 \mathrm{C}>\mathrm{T}, \mathrm{c} .953 \mathrm{~T}>\mathrm{G}$ and c. $757 \mathrm{C}>\mathrm{T}(14)$. Among these, the mutations that have been commonly observed in particular ethnicities are: $c .120+1 \mathrm{G}>\mathrm{A}$ (Tunisian- $91 \%$ ), c.757C > T (Pakistani- 89\%), c.953T > G (Chinese-58\%) c.1171A > G (64\%) and c.337A > T (52\%) in the Irish population. Thus, the prevalence of certain alleles in particular ethnicities is likely to be due to founder effects.

Bidchol et al. (15) were the first to report the mutation spectrum of the GALNS gene in Indian Morquio-A syndrome patients. In that study, the patients studied were primarily from the Northern and Southern regions of the country. Nonetheless, considering the diverse population of India with different ethnicities, the scope for better diagnosis and management of Morquio-A syndrome would be enhanced if the mutation spectrum is known specifically for the individual ethnicities.

In present study we aimed to identify the mutation spectrum in Gujarati Indian patients with Morquio-A syndrome.

\section{Materials And Methods}

\section{Study subjects and ethics statement}

The study was carried out in 25 children from Gujarat-India, affected with dysostosis multiplex as per the clinical diagnosis. The age range of the subjects was 11 months to 21 years. Clinical details were noted in a case record form and an informed consent was obtained from each family. The study protocol was approved by Institutional Ethics committee of FRIGE, Ahmedabad (Reg No- E/13237) as per the Helsinki declaration. $5 \mathrm{ml}$ peripheral blood was collected from the patients for leucocyte enzyme assay and isolation of DNA.

Urinary GAG Testing and Leucocyte Enzyme Assay

Page $2 / 14$ 
Urinary GAG quantitative study was performed by dimethyl methylene blue (DMB) dye method (16). The concentration of urine creatinine of individual patients was measured by a biochemistry analyzer using a commercial kit and expressed in $\mathrm{mg} / \mathrm{mmol}$ of creatinine. The pretreatment of urine prior to qualitative analysis of GAG by electrophoresis, was performed by a method described by Hopwood and Harrison, 1982 (17). Lysosomal enzyme $\beta$-galactose-6-sulfate-sulfatase activity was carried out from leucocytes using fluorogenic synthetic substrate 4methylumbelliferyl- $\beta$-galactose-6-sulfate-triethyl ammonium (18). The fluorescence of free 4-methylumbelliferone (4 MU) was measured by a spectrofluorometer (LS55-Perkin Elmer) to determine the $\beta$-galactose-6-sulfate-sulfatase activity. The enzyme activity was expressed as the amount of substrate in nmol cleaved per hour per $\mathrm{mg}$ of protein in the cell lysates.

\section{Molecular characterization}

For all enzymatically confirmed cases of Morquio-A syndrome, genomic DNA was isolated from peripheral blood by the standard salting out method (19). The exonic regions and the flanking intronic sequences of the GALNS gene were amplified by PCR in 14 fragments using prescribed primers (See Additional file 1). A 10 $\mu$ reaction mixture for the DNA amplification of each fragment was made up of 100ng genomic DNA, $1 \mathrm{mM}$ dNTPs, and 10XPCR buffer. 30 cycles of amplification were performed, each consisting denaturation at $94 \llbracket \mathrm{C}$ for 1 minute, annealing at $60-65 \llbracket \mathrm{C}$ suitable for each exon for 45 seconds and extension at $72 \varangle \mathrm{C}$ for 45 seconds in a thermal cycler. The final extension time was of 10 minutes. PCR products along with the appropriate negative controls and a 100 base-pair DNA ladder were then subjected to electrophoresis in $2 \%$ agarose gel and purified using Exo-SAP-IT ${ }^{\mathrm{TM}}$ (USB Corporation, $\mathrm{OH}, \mathrm{USA}$ ). The purified products were sequenced using BigDye Terminator $\mathrm{V} 3.1$ and capillary electrophoresis was performed using an automated sequencer ABI-3500 (Applied Biosystems, CA, USA) for mutation analysis of GALNS. The sequences through the bidirectional sequencing was analyzed by comparing with the reference sequence of GALNS (RefSeq cDNA NM_000512.5). The mutations identified were annotated with Human Gene Mutation Database, dbSNP and McGill University database

\section{Bioinformatic analysis}

The functional effects of non-synonymous single nucleotide substitution (nsSNPs) were predicted using software SIFT (Sorting Intolerant From Tolerant) (http://sift.jcvi.org), Polyphen2 (Polymorphism Phenotyping v2) (http://genetics.bwh.harvard.edu/pph2/), MutPred (http://mutpred.mutdb.org/) and PROVEAN (http://provean.jcbi.org). The pathogenicity of indels were confirmed by MutationTaster (http://www.mutationtaster.org/). Evolutionary conservation of amino acid residues of GALNS was analyzed using ClustalW program (http://www.uniprot.org/help/sequence-alignments).

Haplotype analysis was performed in all Morquio-A syndrome patients using microsatellite markers D16S3121, D16S3026 and D16S3023 (Figure 1) and SNPs. To confirm the results obtained from haplotype analysis, 200 normal unrelated individuals were screened using ARMS-PCR for P77R variant (See Additional file 1). A reaction mixture of $13 \mu$ l was prepared by adding the components in their required quantity that included Emerald master mix from Takara (10 $\mu \mathrm{l} /$ tube); distilled water $(2 \mu \mathrm{l} /$ tube) and 5pmol common reverse primer $(1 \mu \mathrm{l} /$ tube). Normal forward and mutant forward primers $(1 \mu \mathrm{l} /$ tube) and 100ng of DNA was added along with the reaction mixture to the respective PCR tubes. The PCR was conducted as per the required condition such as, initial denaturation at $94^{\circ} \mathrm{C}$ for 5 minutes of 1 cycle followed by 30 cycles of denaturation at $94^{\circ} \mathrm{C}$ for 30 seconds, annealing (normal primer $-50^{\circ} \mathrm{C}$, mutant primer $-61^{\circ} \mathrm{C}$ ) for 45 seconds, elongation at $72^{\circ} \mathrm{C}$ for 1 minute and the final elongation was at $72^{\circ} \mathrm{C}$ for 5 minutes of 1 cycle. Amplified products were separated on $2 \%$ agarose gel at $100 \mathrm{~V}$ for 30 minutes and viewed on UV image analyzer.

\section{Results}

The present study included 25 Gujarati Indian patients clinically and enzymatically diagnosed as Morquio-A. Seven of the 25 patients had consanguineous parents and remaining 18 patients were endogamous. The age of the patients at diagnosis ranged from 11 months to 21 years with a mean age of 4.32 years. The detailed information about the clinical features of all the patients is provided in Table 1 . The clinical features present in all the patients included short stature, short fingers and short neck-trunk, and frontal bossing. Kyphosis was seen in $10 / 25$ patients and platyspondyly in 5/25 patients. The other prominent symptoms associated with Morquio-A were observed with a low frequency in the present study such as corneal clouding was seen only in 1/20 (5.00\%), knock knee in 6/25 (24.00\%) patients. On radiological assessment most of them showed anterior beaking of vertebral bodies, acetabular dysplasia, platyspondyly and multiple dysplastic epiphyses. 
Table 1

Clinical, biochemical and molecular details of the Gujarati Patel patients with Morquio-A syndrome

\begin{tabular}{|c|c|c|c|c|c|c|c|c|c|}
\hline $\begin{array}{l}\text { Sr. } \\
\text { No }\end{array}$ & $\begin{array}{l}\text { Case } \\
\text { ID }\end{array}$ & $\begin{array}{l}\text { Age at } \\
\text { diagnosis }\end{array}$ & Sex & $\begin{array}{l}\text { Religion } \\
\text { / caste }\end{array}$ & Consanguinity & Clinical features & $\begin{array}{l}\text { Enzyme } \\
\text { activity } \\
\text { (nmol/hr/mg) }\end{array}$ & Variant & Zygosity \\
\hline 1 & $\mathrm{P} 1$ & 3 years & $\mathrm{F}$ & $\begin{array}{l}\text { Hindu } \\
\text { Patel }\end{array}$ & No & $\begin{array}{l}\text { Kyphosis, Anterior } \\
\text { beaking of bones, } \\
\text { Short fingers }\end{array}$ & 6.0 & $\begin{array}{l}\text { p.P77R } \\
\text { c. } 230 \mathrm{C}>\mathrm{G}\end{array}$ & Homozygous \\
\hline 2 & $\mathrm{P} 2$ & 1.9 years & $M$ & $\begin{array}{l}\text { Hindu } \\
\text { Patel }\end{array}$ & No & $\begin{array}{l}\text { Anterior offset of } \\
\text { D1-14 relation to Da } \\
\text { vertebral body, } \\
\text { exaggerated lumbar } \\
\text { lordosis with mild } \\
\text { kyphotic curvature } \\
\text { at dorsa-lumbar } \\
\text { junction, anterior } \\
\text { inferior beaking of } \\
\text { lower dorsal and } \\
\text { lumbar vertebral } \\
\text { body }\end{array}$ & 3.7 & $\begin{array}{l}\text { p.P77R } \\
\text { c. } 230 \mathrm{C}>\mathrm{G}\end{array}$ & Homozygous \\
\hline 3 & P3 & $\begin{array}{l}11 \\
\text { months }\end{array}$ & $\mathrm{F}$ & Muslim & Yes & $\begin{array}{l}\text { Kyphotic deformity } \\
\text { in lower thoracic } \\
\text { and upper lumbar } \\
\text { spine with gibbous } \\
\text { formation at D11- } \\
\text { D12, minimal } \\
\text { scoliosis with } \\
\text { convexity towards } \\
\text { lumbar side }\end{array}$ & 0.75 & $\begin{array}{l}\text { p.P77R } \\
\text { c. } 230 \mathrm{C}>\mathrm{G}\end{array}$ & Homozygous \\
\hline 4 & P4 & 1.5 years & $M$ & Muslim & Yes & Skeletal dysplasia & 11.1 & $\begin{array}{l}\text { p.L36R } \\
\text { c. } 107 T>\text { G }\end{array}$ & Homozygous \\
\hline 5 & P5 & 8 years & $\mathrm{F}$ & $\begin{array}{l}\text { Hindu } \\
\text { Patel }\end{array}$ & No & $\begin{array}{l}\text { Skeletal deformity } \\
\text { noted at age of 8-9 } \\
\text { months, keeping } \\
\text { neck extended, } \\
\text { knock knee, } \\
\text { kyphosis of back, } \\
\text { delayed motor } \\
\text { development, } \\
\text { frontal bossing, } \\
\text { corneal clouding, } \\
\text { short neck and } \\
\text { trunk, widening of } \\
\text { wrist and elbow, } \\
\text { mild hypotonia }\end{array}$ & 4.8 & $\begin{array}{l}\text { p.P77R } \\
\text { c. } 230 \mathrm{C}>\mathrm{G}\end{array}$ & Homozygous \\
\hline 6 & P6 & 1 year & $\mathrm{F}$ & $\begin{array}{l}\text { Hindu } \\
\text { Soni }\end{array}$ & No & $\begin{array}{l}\text { Skeletal } \\
\text { abnormality, D-L } \\
\text { junction } \\
\text { prominence with } \\
\text { scoliosis, large } \\
\text { skull, frontal } \\
\text { bossing, kyphosis, } \\
\text { mild platyspondyly, } \\
\text { bullet shaped } \\
\text { phalanges, wide } \\
\text { ribs }\end{array}$ & 4.15 & $\begin{array}{l}\text { c.1241_1242insA } \\
\text { (p.1416HfsX2) }\end{array}$ & Homozygous \\
\hline 7 & P7 & 2 years & $M$ & $\begin{array}{l}\text { Hindu } \\
\text { Ahir }\end{array}$ & No & $\begin{array}{l}\text { Short stature, D/L } \\
\text { kyphosis, } \\
\text { hydrocephalus }\end{array}$ & 4.3 & $\begin{array}{l}\text { p.D39G } \\
\text { c. } 116 A>G\end{array}$ & Homozygous \\
\hline 8 & P8 & 6 years & $M$ & Darbar & Yes & $\begin{array}{l}\text { Skeletal dysplasia, } \\
\text { hypo scoliosis, } \\
\text { pigeon chest, knock } \\
\text { knee, rickets }\end{array}$ & 5.6 & $\begin{array}{l}\text { p.P151L } \\
\text { c. } 452 \mathrm{C}>\mathrm{T}\end{array}$ & Homozygous \\
\hline
\end{tabular}

*Another variant remains unidentified, ** Checked only for p.P77R which was absent 


\begin{tabular}{|c|c|c|c|c|c|c|c|c|c|}
\hline $\begin{array}{l}\text { Sr. } \\
\text { No }\end{array}$ & $\begin{array}{l}\text { Case } \\
\text { ID }\end{array}$ & $\begin{array}{l}\text { Age at } \\
\text { diagnosis }\end{array}$ & Sex & $\begin{array}{l}\text { Religion } \\
\text { / caste }\end{array}$ & Consanguinity & Clinical features & $\begin{array}{l}\text { Enzyme } \\
\text { activity } \\
\text { (nmol/hr/mg) }\end{array}$ & Variant & Zygosity \\
\hline 9 & P9 & 2 years & M & $\begin{array}{l}\text { Hindu } \\
\text { Patel }\end{array}$ & No & $\begin{array}{l}\text { Bullet shaped } \\
\text { metacarpals, } \\
\text { platyspondyly, D-L } \\
\text { kyphosis, bilateral } \\
\text { hip dysplasia }\end{array}$ & 7.7 & $\begin{array}{l}\text { p.P77R } \\
\text { c. } 230 \mathrm{C}>\mathrm{G}\end{array}$ & Homozygous \\
\hline 10 & P10 & 1 year & $M$ & $\begin{array}{l}\text { Hindu } \\
\text { Patel }\end{array}$ & No & $\begin{array}{l}\text { DL Kyphosis, } \\
\text { platyspondyly, } \\
\text { abnormal CFE B/L } \\
\text { femur }\end{array}$ & 3.0 & $\begin{array}{l}\text { p.P77R } \\
\text { c. } 230 \mathrm{C}>\mathrm{G}\end{array}$ & Homozygous \\
\hline 11 & P11 & 6 years & $M$ & $\begin{array}{l}\text { Hindu } \\
\text { Patel }\end{array}$ & No & $\begin{array}{l}\text { Short stature, short } \\
\text { neck, short fingers, } \\
\text { skeletal dysplasia }\end{array}$ & 1.1 & $\begin{array}{l}\text { P77R } \\
\text { c. } 230 \mathrm{C}>\mathrm{G} \text { and } \\
\text { p. } 6255 \mathrm{~A} \\
\text { c. } 764 \mathrm{G}>\mathrm{C}\end{array}$ & $\begin{array}{l}\text { Compound } \\
\text { heterozygous }\end{array}$ \\
\hline 12 & P12 & 1.7 years & $\mathrm{F}$ & $\begin{array}{l}\text { Hindu } \\
\text { Patel }\end{array}$ & No & $\begin{array}{l}\text { Mild skeletal } \\
\text { abnormality, } \\
\text { sometimes gait } \\
\text { disturbance, mild } \\
\text { coarse face }\end{array}$ & 0.198 & $\begin{array}{l}\text { p.P77R } \\
\text { c. } 230 \mathrm{C}>\mathrm{G}\end{array}$ & Homozygous \\
\hline 13 & P13 & 2.5 years & M & $\begin{array}{l}\text { Hindu } \\
\text { Patel }\end{array}$ & No & $\begin{array}{l}\text { Bullet shaped } \\
\text { phalanges bones, } \\
\text { platyspondyly, } \\
\text { anterior beaking of } \\
\text { vertebral bodies, } \\
\text { distorted } \\
\text { acetabulum, genu } \\
\text { valgum }\end{array}$ & 0.05 & $\begin{array}{l}\text { p.L350P } \\
\text { c. } 1049 T>C\end{array}$ & Homozygous \\
\hline 14 & P14 & 3.7 years & $\mathrm{F}$ & $\begin{array}{l}\text { Hindu } \\
\text { Patel }\end{array}$ & No & $\begin{array}{l}\text { Short stature, genu } \\
\text { valgum, } \\
\text { spondyloepiphyseal } \\
\text { dysplasia, } \\
\text { acetabulum } \\
\text { dysplasia, } \\
\text { epiphyseal } \\
\text { dysplasia }\end{array}$ & 0.37 & $\begin{array}{l}\text { p.P77R } \\
\text { c. } 230 \mathrm{C}>\mathrm{G}\end{array}$ & Homozygous \\
\hline 15 & P15 & 4 years & $\mathrm{F}$ & Hindu & No & $\begin{array}{l}\text { Mild anterior } \\
\text { beaking of vertebral } \\
\text { bodies, short and } \\
\text { wide metacarpals } \\
\text { noted with pointed } \\
\text { base, Madelung } \\
\text { deformity in wrist }\end{array}$ & 0.08 & $\begin{array}{l}\text { c.839_41delACA } \\
\text { and } \\
\text { p.P125L } \\
\text { c. } 374 \mathrm{C}>\mathrm{T}\end{array}$ & $\begin{array}{l}\text { Compound } \\
\text { Heterozygous }\end{array}$ \\
\hline 16 & P16 & 12 years & $\mathrm{F}$ & Hindu & No & $\begin{array}{l}\text { Dysostosis } \\
\text { multiplex, deep } \\
\text { acetabula, Varus } \\
\text { deformity of upper } \\
\text { lip }\end{array}$ & 3.4 & $\begin{array}{l}\text { p.C79R } \\
\text { c. } 235 T>C\end{array}$ & Homozygous \\
\hline 17 & P17 & 21 years & $M$ & $\begin{array}{l}\text { Hindu } \\
\text { Patel }\end{array}$ & No & Skeletal dysplasia & 0.03 & $\begin{array}{l}\text { p.P77R } \\
\text { c. } 230 \mathrm{C}>\mathrm{G}\end{array}$ & Homozygous \\
\hline
\end{tabular}

*Another variant remains unidentified, ** Checked only for p.P77R which was absent 


\begin{tabular}{|c|c|c|c|c|c|c|c|c|c|}
\hline $\begin{array}{l}\text { Sr. } \\
\text { No }\end{array}$ & $\begin{array}{l}\text { Case } \\
\text { ID }\end{array}$ & $\begin{array}{l}\text { Age at } \\
\text { diagnosis }\end{array}$ & Sex & $\begin{array}{l}\text { Religion } \\
\text { / caste }\end{array}$ & Consanguinity & Clinical features & $\begin{array}{l}\text { Enzyme } \\
\text { activity } \\
\text { (nmol/hr/mg) }\end{array}$ & Variant & Zygosity \\
\hline 18 & P18 & 5 years & $M$ & Javia & No & $\begin{array}{l}\text { Skeletal dysplasia, } \\
\text { bilateral genu } \\
\text { valgum, ankle } \\
\text { deformity, bilateral } \\
\text { Plano valgus feet, } \\
\text { spondylo } \\
\text { epiphyseal } \\
\text { dysplasia }\end{array}$ & 0.25 & $\begin{array}{l}\text { p.P77R } \\
\text { c. } 230 \mathrm{C}>\mathrm{G}\end{array}$ & Homozygous \\
\hline 19 & P19 & 3.5 years & M & Muslim & Yes & $\begin{array}{l}\text { Dysmorphic face, } \\
\text { dysostosis } \\
\text { multiplex, short } \\
\text { stature }\end{array}$ & 0.04 & $\begin{array}{l}\text { p.L36R } \\
\text { c.107T > G }\end{array}$ & Homozygous \\
\hline 20 & P20 & 4 years & $\mathrm{F}$ & Muslim & Yes & $\begin{array}{l}\text { Short stature, } \\
\text { difficulty in } \\
\text { standing from } \\
\text { sitting, spine } \\
\text { lumbar lordosis, } \\
\text { wrist broadening }\end{array}$ & 0.8 & c. $121-7 C>G$ & Homozygous \\
\hline 21 & P21 & 1.6 years & M & $\begin{array}{l}\text { Hindu } \\
\text { Patel }\end{array}$ & No & $\begin{array}{l}\text { Mild scoliosis, mild } \\
\text { prominence of } \\
\text { cerebral sulci \& } \\
\text { both lateral } \\
\text { ventricles, inferior } \\
\text { beaking of lower } \\
\text { dorsal \& lumbar } \\
\text { vertebra, kyphotic } \\
\text { deformity, relatively } \\
\text { short metacarpals } \\
\text { with pointed } \\
\text { proximal ends }\end{array}$ & 0.02 & $\begin{array}{l}\text { p.P77R } \\
\text { c. } 230 \mathrm{C}>\mathrm{G}\end{array}$ & Homozygous \\
\hline 22 & P22 & 4.6 years & $M$ & $\begin{array}{l}\text { Hindu } \\
\text { Patel }\end{array}$ & No & $\begin{array}{l}\text { Short stature, } \\
\text { frontal bossing, } \\
\text { pectus carinatum, } \\
\text { genu valgum, } \\
\text { kyphoscoliosis }\end{array}$ & 0.04 & Not determined & $-\star \star \star$ \\
\hline 23 & P23 & 4 years & $\mathrm{F}$ & $\begin{array}{l}\text { Hindu } \\
\text { Patel }\end{array}$ & No & $\begin{array}{l}\text { Short stature, wrist } \\
\text { deformity, mild pes } \\
\text { carinatum, } \\
\text { platyspondyly, } \\
\text { anterior beaking of } \\
\text { all vertebrae, } \\
\text { bilateral acetabular } \\
\text { dysplasia, knocked } \\
\text { knees }\end{array}$ & 0.09 & $\begin{array}{l}\text { p.P77R } \\
\text { c. } 230 \mathrm{C}>\mathrm{G}\end{array}$ & Heterozygous* \\
\hline 24 & P24 & 6 years & $\mathrm{F}$ & Muslim & Yes & $\begin{array}{l}\text { Chest deformity, } \\
\text { bending of knees, } \\
\text { coarse face, } \\
\text { increased wrist } \\
\text { angle, knock knee, } \\
\text { flat foot, pectus } \\
\text { carinatum }\end{array}$ & 0.025 & $\begin{array}{l}\text { p.P77R } \\
\text { c. } 230 \mathrm{C}>\mathrm{G}\end{array}$ & Homozygous \\
\hline 25 & P25 & 2 years & $\mathrm{F}$ & Muslim & Yes & Skeletal dysplasia & 0.025 & $\begin{array}{l}\text { p.P77R } \\
\text { c. } 230 \mathrm{C}>\mathrm{G}\end{array}$ & Homozygous \\
\hline
\end{tabular}

The urinary GAG analysis and the GALNS enzyme activity were determined for all the patients. Elevated levels of urinary GAG were observed in all the patients with excess of KS and C6S by 1D electrophoresis. The enzyme activity of $\mathrm{N}$-acetylgalactosamine-6sulfate sulfatase in all the patients was in the range of $0.02-11.1 \mathrm{nmol} / \mathrm{hour} / \mathrm{mg}$ of protein and the mean enzyme activity was $2.30 \mathrm{nmol} / \mathrm{hour} / \mathrm{mg}$ of protein which was less than $10 \%$ of the mean normal enzyme activity (Table 1 ).

The molecular testing of the GALNS gene has identified 11 mutations in the GALNS gene from the 24 patients (Fig. 2, Table 1). No mutation could be identified in one patient (P22). The mutations found include eight missense mutations (84\%): c.107T > G (p.L36R), c.116A>G (p.D39G), c.230C > G (p.P77R), c.235T > C (p.C79R), c.374C > T (pP125L), c.452C > T (p.P151L), c.764G > C (p.G255A) and c.1049T >C (p.L350P), one splice 
site mutation (4\%): c.121-7C > G, one small insertion (4\%): c.1241_1242insA (p.1416HfsTer2) and one small deletion (4\%): c.839_41delACA. Three missense mutations, one splice site mutation and two indels were novel (p.D39G, p.G255A, p.L350P, c.121-7C > G, c.839_41delACA and c.1241_1242insA/p.1416HfsTer2) (Fig. 2). All the mutations were present in homozygous state except two mutations in compound heterozygous state (p.P77R/p.G255A and c.839_41delACA /p.P125L) and one in heterozygous (p.P77R) state with another variant remaining unidentified.

All the novel mutations were tested for their pathogenicity using pathogenicity prediction tools MutationTaster, MutPred, PolyPhen2, SIFT and PROVEAN. All the three missense mutations were predicted as pathogenic by aforementioned bioinformatics predictive tools. The respective scores and likely basis of effect on the protein for the non-synonymous single nucleotide substitutions found are presented in Table 2 . The pathogenicity of the novel splice site mutation and small indels was confirmed by MutationTaster. The sequence alignment and ortholog of GALNS are highly conserved among various species for the residues D39, P77, C79, P125, P151, G255, L350, in which missense mutations were found in this study. Additional file 2 gives the details of conservation of GALNS in orthologs (Additional file 2). 
Table 2

Output of in silico analysis of GALNS missense variants detected in Morquio-A patients

\begin{tabular}{|c|c|c|c|c|c|c|c|c|}
\hline $\begin{array}{l}\text { Sr. } \\
\text { No. }\end{array}$ & $\begin{array}{l}\text { Exon/ } \\
\text { Intron }\end{array}$ & $\begin{array}{l}\text { Nucleotide } \\
\text { Change }\end{array}$ & $\begin{array}{l}\text { Change } \\
\text { in } \\
\text { Protein }\end{array}$ & $\begin{array}{l}\text { Type of } \\
\text { variant }\end{array}$ & MutPred analysis for missense variants & $\begin{array}{l}\text { PolyPhen- } \\
2 \text { analysis }\end{array}$ & $\begin{array}{l}\text { SIFT } \\
\text { result }\end{array}$ & $\begin{array}{l}\text { PROVEAN } \\
\text { (cutoff=-2.5) }\end{array}$ \\
\hline \multirow[t]{2}{*}{1.} & \multirow{4}{*}{$\begin{array}{l}\text { Exon } \\
1\end{array}$} & \multirow[t]{2}{*}{ c. $107 \mathrm{~T}>\mathrm{G}$} & \multirow[t]{2}{*}{ p.L36R } & \multirow[t]{2}{*}{ Missense } & 0.859 & 1 & 0 & -3.555 \\
\hline & & & & & $\begin{array}{l}\text { Loss of stability, Gain of methylation at } \\
\text { L36, Loss of loop, Gain of disorder, Loss } \\
\text { of sheet }\end{array}$ & $\begin{array}{l}\text { Probably } \\
\text { damaging }\end{array}$ & Damaging & Deleterious \\
\hline \multirow[t]{2}{*}{2.} & & \multirow[t]{2}{*}{ c. $116 A>G$} & \multirow[t]{2}{*}{ p.D39G } & \multirow[t]{2}{*}{ Missense } & 0.823 & 0.997 & 0 & -5.863 \\
\hline & & & & & $\begin{array}{l}\text { Loss of stability, Gain of catalytic residue } \\
\text { at D39, Loss of sheet, Gain of disorder, } \\
\text { Loss of helix }\end{array}$ & $\begin{array}{l}\text { Probably } \\
\text { damaging }\end{array}$ & Damaging & Deleterious \\
\hline \multirow[t]{2}{*}{3.} & \multirow{4}{*}{$\begin{array}{l}\text { Exon } \\
2\end{array}$} & \multirow[t]{2}{*}{ c. $230 \mathrm{C}>\mathrm{G}$} & \multirow[t]{2}{*}{ p.P77R } & \multirow[t]{2}{*}{ Missense } & 0.948 & 1 & 0.01 & -4.973 \\
\hline & & & & & $\begin{array}{l}\text { Gain of methylation at P77, Gain of } \\
\text { MoRF binding, Gain of phosphorylation, } \\
\text { Gain of catalytic residue at S82, Loss of } \\
\text { disorder }\end{array}$ & $\begin{array}{l}\text { Probably } \\
\text { damaging }\end{array}$ & Damaging & Deleterious \\
\hline \multirow[t]{2}{*}{4.} & & \multirow[t]{2}{*}{ c. $235 \mathrm{~T}>\mathrm{C}$} & \multirow[t]{2}{*}{ p.P79R } & \multirow[t]{2}{*}{ Missense } & 0.965 & 1 & \multirow{2}{*}{$\begin{array}{l}0 \\
\text { Damaging }\end{array}$} & -10.060 \\
\hline & & & & & $\begin{array}{l}\text { Altered Metal binding; Gain of Catalytic } \\
\text { site at C79; Altered Ordered interface; } \\
\text { Gain of Intrinsic disorder; Loss of } \\
\text { Allosteric site at R83 }\end{array}$ & $\begin{array}{l}\text { Probably } \\
\text { damaging }\end{array}$ & & Deleterious \\
\hline \multirow[t]{2}{*}{5.} & \multirow[t]{2}{*}{$\begin{array}{l}\text { Exon } \\
4\end{array}$} & \multirow[t]{2}{*}{ c. $374 \mathrm{C}>\mathrm{T}$} & \multirow[t]{2}{*}{ p.P125L } & \multirow[t]{2}{*}{ Missense } & 0.907 & 1 & \multirow[t]{2}{*}{0.06} & -4.654 \\
\hline & & & & & $\begin{array}{l}\text { Altered Ordered interface; Altered Metal } \\
\text { binding; Gain of Helix; Loss of Relative } \\
\text { solvent accessibility; Loss of Acetylation } \\
\text { at; Altered DNA binding; Loss of Allosteric } \\
\text { site at L128; Loss of Methylation at K130 }\end{array}$ & $\begin{array}{l}\text { Probably } \\
\text { damaging }\end{array}$ & & Deleterious \\
\hline \multirow[t]{2}{*}{6.} & \multirow{2}{*}{$\begin{array}{l}\text { Exon } \\
5\end{array}$} & \multirow[t]{2}{*}{ c. $452 \mathrm{C}>\mathrm{T}$} & \multirow[t]{2}{*}{ p.P151L } & \multirow[t]{2}{*}{ Missense } & 0.920 & 1 & 0 & -8.970 \\
\hline & & & & & $\begin{array}{l}\text { Loss of disorder, Loss of ubiquitination at } \\
\text { K153, Gain of loop, Loss of methylation } \\
\text { at K153, Gain of MoRF binding }\end{array}$ & $\begin{array}{l}\text { Probably } \\
\text { damaging }\end{array}$ & Damaging & Deleterious \\
\hline \multirow[t]{2}{*}{7.} & \multirow{2}{*}{$\begin{array}{l}\text { Exon } \\
8\end{array}$} & \multirow[t]{2}{*}{ c. $764 \mathrm{G}>\mathrm{C}$} & \multirow[t]{2}{*}{ p.G255A } & \multirow[t]{2}{*}{ Missense } & 0.857 & 1 & 0.11 & -5.881 \\
\hline & & & & & $\begin{array}{l}\text { Loss of Relative solvent accessibility; } \\
\text { Altered Transmembrane protein; Altered } \\
\text { Coiled coil; Loss of Allosteric site at E260 }\end{array}$ & $\begin{array}{l}\text { Probably } \\
\text { damaging }\end{array}$ & & Deleterious \\
\hline \multirow[t]{2}{*}{8.} & Exon & c. 1049T > & p.L350P & Missense & 0.958 & 1 & 0.30 & -4.100 \\
\hline & & & & & $\begin{array}{l}\text { Gain of Relative solvent accessibility; } \\
\text { Altered Metal binding; Altered } \\
\text { Transmembrane protein; Gain of } \\
\text { Allosteric site at T348; Gain of Catalytic } \\
\text { site at T348 }\end{array}$ & $\begin{array}{l}\text { Probably } \\
\text { damaging }\end{array}$ & & Deleterious \\
\hline $\begin{array}{l}\text { Effe } \\
\text { red. } \\
\text { con } \\
\text { beir } \\
\text { an }\end{array}$ & of the & $\begin{array}{l}\text { nesis" for a } \\
\text { damaging". }\end{array}$ & ts on the & ucture and & $\begin{array}{l}\text { unction of GALNS, as predicted by use of bi } \\
\text { n predictions by all the tools used. In MutPr } \\
\text { isease. In PolyPhen-2, the scores for neutra } \\
\text { it is "damaging" if the score is } \leq 0.05 \text { and } t\end{array}$ & $\begin{array}{l}\text { nformatics t } \\
\text { d, probability } \\
\text { o damaging } \\
\text { erated if the }\end{array}$ & $\begin{array}{l}\text { ols. Novel va } \\
0.75 \text { is con } \\
\text { ange from } 0 \\
\text { core is }>0.0\end{array}$ & $\begin{array}{l}\text { ants are in } \\
\text { dered a "very } \\
1 \text {, with } 1 \\
\text { In PROVEAN, }\end{array}$ \\
\hline
\end{tabular}

Table 3A: Haplotype analysis of founder mutation p.P77R in Gujarati Patel patients with Morquio-A syndrome using microsatellite markers 


\begin{tabular}{|llllll|}
\hline Markers & Genomic position & $\begin{array}{l}\text { Position wrt } \\
\text { GALNS }\end{array}$ & $\begin{array}{l}\text { Length of the marker } \\
\text { (bp) }\end{array}$ & $\begin{array}{l}\text { Range in our population } \\
\text { (bp) }\end{array}$ & $\begin{array}{l}\text { Range in other populations } \\
\text { (bp) }\end{array}$ \\
\hline D16S3121 & $\begin{array}{l}88,455,110- \\
88,455,199\end{array}$ & 575 kb Upstream & 83 & $69-85$ & $79-85$ \\
\hline D16S3026 & $\begin{array}{l}89,426,440- \\
89,426,641\end{array}$ & $569 \mathrm{~kb}$ Upstream & 202 & $202-217$ & $204-210$ \\
\hline D16S3023 & $\begin{array}{l}89,432,214- \\
89,432,296\end{array}$ & $\begin{array}{l}358 \mathrm{~kb} \\
\text { Downstream }\end{array}$ & 90 & $83-105$ & $87-97$ \\
\hline
\end{tabular}

Table 3B: Haplotype analysis of founder mutation P.P77R in Gujarati Patel patients with Morquio-A syndrome using SNPs.

\begin{tabular}{|c|c|c|c|}
\hline Variation (db SNP ID) & Exon/ Intron & Genomic position & Alleles \\
\hline rs8062286 & Intron 1 & 10873 & $C>T$ \\
\hline rs35588597 & Intron 1 & 10902 & delG \\
\hline Novel & Intron 1 & 11441 & $C>A$ \\
\hline rs12709105 & Intron 1 & 11524 & $C>T$ \\
\hline rs12598981 & Intron 1 & 12339 & $C>A$ \\
\hline rs12444242 & Intron 1 & 17332 & $G>A$ \\
\hline rs12447646 & Intron 1 & 17551 & $C>T$ \\
\hline CM950524 & Exon 2 & 19247 & $C>G$ \\
\hline rs71395332 & Intron 2 & 19347 & $G>A$ \\
\hline rs377453859 & Intron 2 & 19941 & $\mathrm{G}>\mathrm{C}$ \\
\hline rs8054994 & Intron 4 & 21092 & $C>A$ \\
\hline rs12444543 & Intron 5 & 25595 & $\mathrm{~T}>\mathrm{C}$ \\
\hline rs2269333 & Intron 6 & 25857 & $\mathrm{~T}>\mathrm{C}$ \\
\hline rs2269334 & Intron 6 & 25891 & $A>G$ \\
\hline rs2269335 & Intron 6 & 25904 & $C>A$ \\
\hline rs12934499 & Intron 6 & 26099 & $G>A$ \\
\hline rs1064315 & Exon 7 & 26192 & $C>T$ \\
\hline rs11076721 & Intron 7 & 26525 & $A>T$ \\
\hline rs3743546 & Intron 9 & 34959 & $\mathrm{~T}>\mathrm{C}$ \\
\hline rs3859024 & Intron 10 & 35362 & $\mathrm{G}>\mathrm{C}$ \\
\hline rs747232 & Intron 11 & 37349 & $G>A$ \\
\hline rs2303271 & Exon 13 & 43909 & $\mathrm{G}>\mathrm{A}$ \\
\hline rs11076716 & Intron 13 & 47410 & $G>C$ \\
\hline rs11076715 & 3' UTR & 47564 & $G>A$ \\
\hline rs1141390 & 3' UTR & 47895 & $T>C$ \\
\hline rs1135364 & 3' UTR & 48139 & $A>G$ \\
\hline rs1135366 & 3' UTR & 48180 & $A>G$ \\
\hline rs77936719 & 3' UTR & 48229 & $C>G$ \\
\hline
\end{tabular}


We found five previously known missense mutations (p.L36R, p.P77R, p.C79R, p.P125L, p.P151L) (14, 15, 20-22). Out of these, the two missense variants p.P77R and p.C79R have been reported in Indian patients (15), whereas the remaining variants were reported from Mexican, Japanese and Italian population. The proportion of p.L36R in our cohort was $8 \%$ and that of p.P77R was $60 \%$. This indicates the higher prevalence of p.P77R in our study population, specifically in the Patel community. This high frequency of the variant in the population is likely to be due to the founder effect. In order to confirm this, we carried out haplotype analysis in patients using dinucleotide microsatellite markers. The results for the same is depicted in Table 3A. Also, all the patients with p.P77R showed a unique identical genotype for the 28 SNP alleles investigated in the present study (Table 3B). A further study comprising 200 unrelated normal Gujarati individuals was carried out to check for the presence of this variant in the population using ARMS-PCR. None of the individuals showed the presence of the variant except for two individuals who were found to be in carrier state. This data also provides evidence that the p.P77R variant is a founder mutation for Morquio-A syndrome especially in Patel community of Gujarati Indians.

\section{Discussion}

The present study is the first to report the clinical and genetic profiles of patients with Morquio-A syndrome in Indian Gujarati patients. Our study cohort consisted of 25 patients with consanguinity in $28 \%$ and endogamy in the remaining. A previous study by Bidchol et al (15) was also carried out in Indian patients but mainly comprising northern and southern region of the country and none from Gujarat.

The mean age at the first symptom was 4.32 years. The mean age reported in various studies differ across populations with Bidchol et al (15) reporting a higher mean age of 6.7 years at presentation, whereas, Montano et al (23) and Lin et al (24) have reported a lower mean age of 2.1 years and 2.0 years respectively. This depends on awareness among clinicians, patients and availability of a diagnostic facility. In the present study, majority of the referrals were from pediatric orthopedic specialists. Use of urine GAG electrophoresis with high excretion of KS with CS seems to be the reason for early diagnosis.

The clinical phenotype of the patients consisted of marked features like short stature, short fingers to kyphosis, scoliosis, genu valgum and skeletal dysplasia. Leong et al (12) reported such severe phenotypes in all the Malaysian patients with Morquio A syndrome. A similar phenotypic spectrum was reported in Indian Morquio-A syndrome patients (15) where short stature, genu valgum and pectus carinatum were the most commonly observed. The International Morquio-A syndrome registry has given the 5 most common initial symptoms in Morquio-A syndrome to be short stature (49.9\%), genu valgum (45.1\%), kyphosis (44.4\%), pectus carinatum (43.6\%), and abnormal gait (37.8\%). In this study we observed short stature (100\%), genu valgum (32\%) and kyphosis (40\%). We found, mild kyphosis and scoliosis were common at an early age, as is expected. However, serious skeletal abnormality and dysostosis multiplex was observed in patients at an advanced age. These findings support the fact that the phenotypic severity increases with advancement of age as reviewed (25).

All the patients had persistent increased urine excretion of GAGs predominantly KS with or without HS (heparan sulphate). The similar observation has been reported in Morquio-A syndrome patients (3). This clearly indicates the use of urinary GAG electrophoresis as the first line screening test and as a differential diagnosis of Morquio-A syndrome. The GALNS enzyme activity in the KS positive patients ranged from undetectable to less than $10 \%$ of the total enzyme activity. Our results are consistent with that observed by Leong et al (12) in the Malaysian population and other reported studies $(15,26,27)$.

On mutational analysis of the GALNS gene in the biochemically diagnosed Morquio-A syndrome patients, we found that out of the 14 exons, exons 1, 2, 5 and 11 harbor most mutations. The study by Bidchol et al (15) showed exon 8 to harbor most of the mutations followed by exon 1 and 7. This is in contrast to our observation of exon 2 (p.P77R) being the hot spot site for mutation. This is likely to be due to absence of Gujarati patients in the earlier study from India.

Our study has identified a total of eleven mutations, out of which eight are missense mutations. This finding is similar to that reported by Bidchol et al (15) as well as matches with the trend observed in HGMD, where for Morquio A, maximum missense mutations have been reported followed by other types. Five out of eight missense variants in our study have been previously reported. None of the ten most common mutations as per the earlier report (3) were found in our study. This suggests that there is heterogeneity at the molecular level. Thus, we can infer that mutation spectrum is influenced by ethnicity. One of the missense variants (p.P77R) more frequently seen in our study has been reported earlier to be present in Indian patient (20) with unknown ethnicity. This mutation was also reported being linked to severe phenotype (28), indicating the association of the variant with severe phenotype in Indian patients in the present study. Bidchol et al (15) has also reported a mutation at the same position (p.P77S), suggesting that the proline at position 77 is important for the enzyme activity and any change can lead to clinical manifestations of Morquio-A syndrome. As reported by Sukegawa (29), proline at 77 has a key role in preventing the internalization in hydrophobic patch of the protein. The positively charged side chain of arginine instead of proline is expected to have a destabilizing effect in this process. The missense variant p.L36R was also found in two patients. This variant has been previously reported by Tomatsu et al (30) and Morrone et al (14) indicating its association with attenuated phenotype. Another missense variant p.P151L reported in this study was also reported previously by Tomatsu et al (20). Proline is conserved at 151 position, thereby any substitution at this site will lead to alteration in 
packing of protein (29). The missense variant p.C79R was first reported by Bidchol et al (15) in an Indian patient. Our study also reports this variant in one patient in homozygous condition. The missense variant p.P125L was found in a patient in compound heterozygous condition. This variant has been reported earlier by Tomatsu et al (21) in Japanese population showing severe phenotype for Morquio-A.

Our study has also identified 6 novel mutations that include 3 missense mutations, 1 splice site, 1 small insertion and 1 small deletion. The novel missense mutation p.D39G is an active site mutation which was found in one patient (P7). The noticeable phenotype was short stature, kyphosis and hydrocephalus. Morrone et al (14) and Tuyuz et al (28) have reported p.D40N to be associated with severe phenotype in Morquio-A syndrome patient. The other two novel missense variants p.G255A and p.L350P were found in compound heterozygous and homozygous conditions respectively. The splice site variant c.121-7 C > G identified in the present study has not been reported till date. The patient with this mutation presented with X-ray showing spine lumbar lordosis and wrist broadening. Generally, mutations in the splice site region leads to the formation of abnormal protein due to splicing defect. In our study, we have also identified a small insertion p.1416 HfsTer2 in one patient which is a novel mutation. The prominent clinical features of skeletal abnormality, kyphoscoliosis, frontal bossing and platyspondyly were observed in this patient. A small deletion in exon 8 was found in a patient in compound heterozygous condition. As per the data by Tomatsu et al (30), 20 small deletions have been reported in the GALNS gene. The deletion in the present study was not previously reported in Indian population.

A set of bioinformatics tools have predicted the pathogenicity of the novel mutations by in silico analysis as described earlier. And all of them were at the conserved regions and mutations at these positions are highly likely to be pathogenic and causative of Morquio-A syndrome.

Interestingly, p.P77R was predominantly found in the Patel community of Gujarat. In order to confirm the founder effect, haplotype study was carried out in unrelated individuals of the same ethnicity using dinucleotide microsatellite markers. All the tested individuals were negative for this mutation except two individuals showing a carrier status. This confirms that p.P77R is a founder mutation in the Gujarati Patel population. Our study further indicates the reason of significant burden of rare diseases in India as has been observed recently (31). A greater attention needs to be paid to the different subpopulations and the effect of rare disease on community health needs to be elucidated.

\section{Conclusion}

The mutation spectrum for Morquio-A syndrome patients of Gujarati Indians is unique, wherein P77R is likely to be the founder mutation in Gujarati Patel ethnicity. This can be further utilized as a molecular marker and for carrier screening of the aforementioned population.

\section{List Of Abbreviations}

GAG: glycosaminoglycans, KS: keratan sulfate, C6S: chondroitin-6-sulfate, ARMS-PCR: amplification refractory mutation system PCR, HS: heparan sulphate

\section{Declarations}

\section{Ethics approval and consent to participate}

- Present study under submission has been approved by the institutional ethics committee [FRIGE's Institute of Human Genetics] wide approval number FRIGE/IEC/5/2010 dated $7^{\text {th }}$ March, 2010. This process is in accordance with the Helsinki declaration.

- An informed consent was obtained from the parents before enrolling the patients for the investigations [This was in accordance with the requirement of the institutional ethics committee].

- An informed consent for publication was also obtained from the individuals included in the submission [This was in accordance with the requirement of the institutional ethics committee].

\section{Consent for publication}

Informed written consent was obtained from all the participants for publication of their clinical details and/or clinical images. A copy of the written consent is available for review by the editor of this journal.

\section{Availability of data and materials}

The dataset generated and/or analyzed during the current study is submitted in the ClinVar repository. Additional File 3 gives information on the ClinVar accession IDs of all the variants of GALNS gene generated or analyzed in the present study.

\section{Competing interests}

All authors declare that they have no conflict of interests (financial or non-financial). 


\section{Funding}

This work is partially supported by Indian Council of Medical Research (ICMR) Project No.: BMS:54/2010. The funding agency was not directly or indirectly involved in the study design, specimen collection, analysis, interpretation and preparation of the manuscript.

\section{Details of the contributions of individual authors}

Designing the study, carrying out the protocol for enzyme assays and molecular study - JS, RB, CP, FJS, SVN, SS. Preparation of manuscript - JS, AN, FJS, SS. Clinical analysis - PN, MS, MC. Data analysis - JS, RB, SS. Haplotype and in silico studies- NP, PD, VT, RB and SS. JS and SS will act as guarantors of the study.

\section{Acknowledgements}

We thank Dr. Harsh Sheth and Dr. Sunil Trivedi for critical reading and evaluating the manuscript and also, Ms. Satakhi Panda and Ms. Niyati Kapasi for their partial help in preparing the manuscript. We are grateful to all the patients and their families for the support, as without their consent this study would not have been possible. We acknowledge Indian Council of Medical Research (ICMR) for partial support to the work.

\section{References}

1. Matalon R, Arbogast B, Justice P, Brandt IK, Dorfman A. Morquio's syndrome: Deficiency of a chondroitin sulfate N-acetylhexosamine sulfate sulfatase. Biochem Biophys Res Commun. 1974;61(2):759-65.

2. Nakashima Y, Tomatsu S, Hori T, Fukuda S, Sukegawa K, Kondo N, et al. Mucopolysaccharidosis iv a: Molecular cloning of the human Nacetylgalactosamine-6-sulfatase gene (GALNS) and analysis of the 5'-flanking region. Genomics. 1994;20(1):99-104.

3. Peracha H, Sawamoto K, Averill L, Kecskemethy H, Theroux M, Thacker M, et al. Molecular genetics and metabolism, special edition: Diagnosis, diagnosis and prognosis of Mucopolysaccharidosis IVA. Vol. 125, Molecular Genetics and Metabolism. 2018. p. 18-37.

4. Hendriksz CJ, Harmatz P, Beck M, Jones S, Wood T, Lachman R, et al. Review of clinical presentation and diagnosis of mucopolysaccharidosis IVA. Mol Genet Metab. 2013;110(1-2):54-64.

5. Theroux MC, Nerker T, Ditro C, MacKenzie WG. Anesthetic care and perioperative complications of children with Morquio syndrome. Paediatr Anaesth. 2012;22(9):901-7.

6. Rodriguez ME, Mackenzie WG, Ditro C, Miller TL, Chidekel A, Shaffer TH. Skeletal dysplasias: Evaluation with impulse oscillometry and thoracoabdominal motion analysis. Pediatr Pulmonol. 2010;45(7):679-86.

7. Solanki GA, Martin KW, Theroux MC, Lampe C, White KK, Shediac R, et al. Spinal involvement in mucopolysaccharidosis IVA (MorquioBrailsford or Morquio A syndrome): Presentation, diagnosis and management. J Inherit Metab Dis. 2013;36(2):339-55.

8. Nelson J. Incidence of the mucopolysaccharidoses in Northern Ireland. Hum Genet. 1997;101(3):355-8.

9. Nelson J, Crowhurst J, Carey B, Greed L. Incidence of the mucopolysaccharidoses in Western Australia. Am J Med Genet. 2003;123A(3):310-3.

10. Meikle PJ, Hopwood JJ, Clague AE, Carey WF. Prevalence of lysosomal storage disorders. J Am Med Assoc. 1999;281(3):249-54.

11. Leadley RM, Lang S, Misso K, Bekkering T, Ross J, Akiyama T, et al. A systematic review of the prevalence of Morquio A syndrome: challenges for study reporting in rare diseases. Orphanet J Rare Dis. 2014;9:173.

12. Leong HY, Abdul Azize NA, Chew HB, Keng WT, Thong MK, Mohd Khalid MKN, et al. Clinical, biochemical and genetic profiles of patients with mucopolysaccharidosis type IVA (Morquio A syndrome) in Malaysia: The first national natural history cohort study. Orphanet $\mathrm{J}$ Rare Dis. 2019;14(1):1-10.

13. The Human Gene Mutation Database. [accessed on 2020 Jul 9]. Available from: http://www.hgmd.org

14. Morrone A, Tylee KL, Al-Sayed M, Brusius-Facchin AC, Caciotti A, Church HJ, et al. Molecular testing of 163 patients with Morquio A (Mucopolysaccharidosis IVA) identifies 39 novel GALNS mutations. Mol Genet Metab. 2014;112(2):160-70.

15. Bidchol AM, Dalal A, Shah H, S S, Nampoothiri S, Kabra M, et al. GALNS mutations in Indian patients with mucopolysaccharidosis IVA. Am J Med Genet A. 2014/09/22. 2014 Nov;164A(11):2793-801. Available from: https://www.ncbi.nlm.nih.gov/pubmed/25252036

16. De Jong JGN, Wevers RA, Laarakkers C, Poorthuis BJHM. Dimethylmethylene blue-based spectrophotometry of glycosaminoglycans in untreated urine: A rapid screening procedure for mucopolysaccharidoses. Clin Chem. 1989;35(7):1472-7.

17. Hopwood JJ, Harrison JR. High-resolution electrophoresis of urinary glycosaminoglycans: an improved screening test for the mucopolysaccharidoses. Anal Biochem. 1982 Jan 1;119(1):120-7. Available from: https://pubmed.ncbi.nlm.nih.gov/6803608

18. van Diggelen OP, Zhao H, Kleijer WJ, Janse HC, Poorthuis BJHM, van Pelt J, et al. A fluorimetric enzyme assay for the diagnosis of Morquio disease type A (MPS IV A). Clin Chim Acta. 1990;187(2):131-9. 
19. Miller SA, Dykes DD, Polesky HF. A simple salting out procedure for extracting DNA from human nucleated cells. Nucleic Acids Res. 1988;16(3):1215.

20. Tomatsu S, Fukuda S, Cooper A, Wraith JE, Rezvi GMM, Yamagishi A, et al. Mucopolysaccharidosis type IVA: Identification of six novel mutations among non-Japanese patients. Vol. 4, Human Molecular Genetics. 1995. p. 741-3.

21. Tomatsu S, Fukuda S, Cooper A, Wraith JE, Ferreira P, Natale P Di, et al. Fourteen novel mucopolysaccharidosis IVA producing mutations in GALNS gene. Hum Mutat. 1997;10(5):368-75.

22. Tomatsu S, Orii KO, Bi Y, Gutierrez MA, Nishioka T, Yamaguchi S, et al. General implications for CpG hot spot mutations: Methylation patterns of the human iduronate-2, sulfatase gene locus. Hum Mutat. 2004;23(6):590-8.

23. Montaño AM, Tomatsu S, Gottesman GS, Smith M, Orii T. International Morquio A Registry: Clinical manifestation and natural course of Morquio A disease. J Inherit Metab Dis. 2007;30(2):165-74.

24. Lin HY, Chuang CK, Chen MR, Chiu PC, Ke YY, Niu DM, et al. Natural history and clinical assessment of Taiwanese patients with mucopolysaccharidosis IVA. Orphanet J Rare Dis. 2014;9(1).

25. Khan S, Alméciga-Díaz CJ, Sawamoto K, Mackenzie WG, Theroux MC, Pizarro C, et al. Mucopolysaccharidosis IVA and glycosaminoglycans. Vol. 120, Molecular Genetics and Metabolism. 2017. p. 78-95.

26. Moreno Giraldo LJ, Escudero Rodríguez ÁM, Sánchez Gómez A, Satizabal Soto JM. Clinical and molecular characteristics of colombian patients with mucopolysaccharidosis IVA, and description of a new galns gene mutation. Mol Genet Metab reports. 2018 Jul 20;16:53-6. Available from: https://www.ncbi.nlm.nih.gov/pubmed/30094185

27. Xie J, Pan J, Guo D, Pan W, Li R, Guo C, et al. Mutation analysis and pathogenicity identification of Mucopolysaccharidosis type IVA in 8 south China families. Gene. 2019;686:261-9. Available from: https://doi.org/10.1016/j.gene.2018.11.051

28. Tüysüz B, Alkaya DU, Toksoy G, Güneş N, Yıldırım T, Bayhan IA, et al. Mutation spectrum and pivotal features for differential diagnosis of Mucopolysaccharidosis IVA patients with severe and attenuated phenotype. Gene. 2019/04/11. 2019 Jul 1;704:59-67. Available from: https://www.ncbi.nlm.nih.gov/pubmed/30980944

29. Sukegawa K. Biochemical and structural analysis of missense mutations in N-acetylgalactosamine-6-sulfate sulfatase causing mucopolysaccharidosis IVA phenotypes. Hum Mol Genet. 2000;9(9):1283-90.

30. Tomatsu S, Montaño AM, Nishioka T, Gutierrez MA, Peña OM, Trandafirescu GG, et al. Mutation and polymorphism spectrum of the GALNS gene in mucopolysaccharidosis IVA (Morquio A). Vol. 26, Human Mutation. 2005. p. 500-12.

31. Consortium Gu, Sivasubbu S, Scaria V. Genomics of rare genetic diseases-experiences from India. Hum Genomics. 2019 Sep 25;14(1):52. Available from: https://www.ncbi.nlm.nih.gov/pubmed/31554517

\section{Figures}

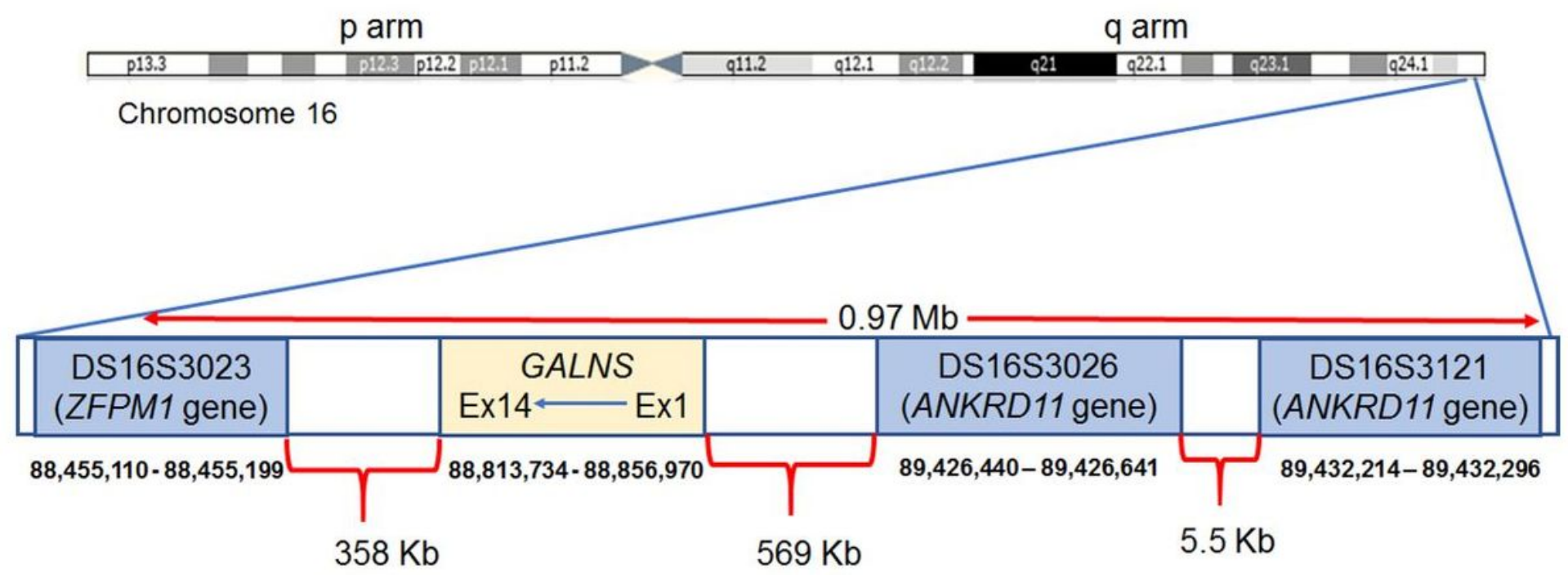

Figure 1

Page 13/14 


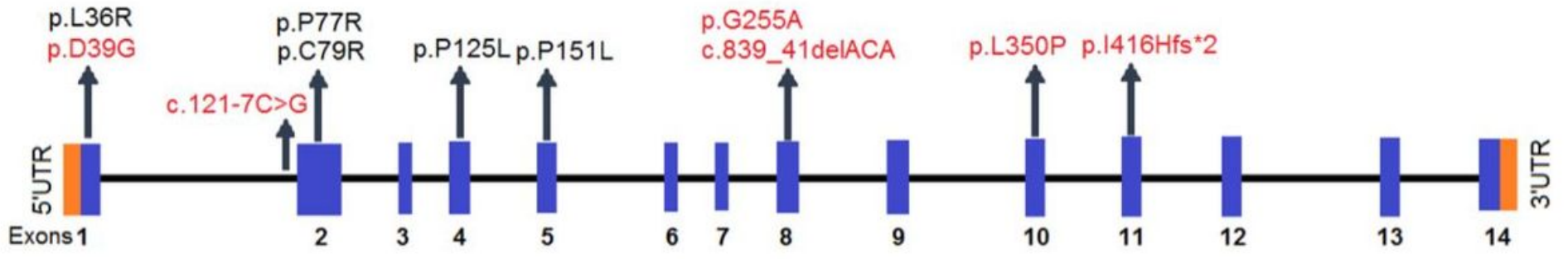

Reported mutations

Novel mutations

\section{Figure 2}

Schematic representation of variants found in GALNS in the current study.

\section{Supplementary Files}

This is a list of supplementary files associated with this preprint. Click to download.

- Additionalfileinformation.docx

- AdditionalFile3.docx

- Additionalfile2.docx

- Additionalfile1.docx 